\title{
Diseño de la Experiencia del Usuario para Espacios Interactivos de Aprendizaje no Formal
}

\author{
Gustavo De la Cruz Martínez, Ana Libia Eslava Cervantes y \\ Ricardo Castañeda Martínez \\ CCADET, UNAM, Circuito Exterior S/N, C.P. 4510, Cd. Universitaria, México, D.F. \\ \{gustavo.delacruz, eslava.cervantes, ricardo.castaneda\}@ccadet.unam.mx
}

\begin{abstract}
Resumen. El diseño de artefactos digitales o espacios interactivos orientados a la educación requiere de metodologías de desarrollo adecuadas que permitan que el producto final favorezca el aprendizaje esperado. En este trabajo se propone utilizar el enfoque del diseño de la experiencia del usuario como guía en el proceso de diseño de espacios interactivos de aprendizaje no formal, ya que una de las principales metas al diseñar este tipo de artefactos digitales es crear una "experiencia" en el usuario que propicie que se alcancen los objetivos de aprendizaje. El objetivo de este trabajo es describir una metodología para el diseño de este tipo de artefactos digitales que vaya más allá de la usabilidad, tomando en cuenta otros aspectos cognitivos, socio-cognitivos y afectivos de la experiencia del usuario al interactuar con estos artefactos, como son: el disfrute de los usuarios, la experiencia estética, el deseo de volver a usar el artefacto, entre otros; todo esto con el fin de favorecer el aprendizaje de acuerdo a los objetivos propios del artefacto. En el trabajo se describe cómo se ha utilizado esta metodología para la construcción de un espacio interactivo orientado a crear una experiencia musical dentro de un museo interactivo dirigido a niños.
\end{abstract}

Palabras clave: Experiencia del usuario, metodología de diseño, aprendizaje no formal.

\section{Introducción}

En [1] se indica que la investigación tradicional en el campo de la Interacción Humano-Computadora ha centrado su estudio en las habilidades y procesos cognitivos del usuario, estudiando principalmente el comportamiento guiado por la racionalidad y dejando de lado la influencia emocional en él. Si bien, la definición de experiencia del usuario aún es discutida en la comunidad de interacción humano-computadora; existe el consenso de que es necesario considerar aspectos más allá de la usabilidad en el diseño de productos o servicios.

Bajo este panorama se propone utilizar el enfoque metodológico del diseño de la experiencia del usuario para complementar el proceso de desarrollo de espacios interactivos orientados al aprendizaje no formal del grupo Espacios y Sistemas Interactivos para la Educación (ESIE) del Centro de Ciencias Aplicadas y Desarrollo Tecnológico de la UNAM. 


\subsection{El Aprendizaje no Formal en Espacios de Difusión de la Ciencia y la Cultura}

En los últimos años ha surgido un gran interés en el tema del aprendizaje que ocurre en modos alternos al que plantea la educación formal. Tanto educadores e investigadores del aprendizaje o del desarrollo cognoscitivo, científicos interesados en la difusión de la ciencia y la cultura, y museólogos han explorado y desarrollado conceptos como el aprendizaje informal, no formal, a lo largo de la vida, entre otros, que si bien comparten muchos elementos en común, sus seguidores hacen hincapié en sus diferencias. Pese a los problemas derivados del costo, instalación, uso, adopción y mantenimiento de las TIC en la educación, la UNESCO sostiene que las TIC pueden mejorar el acceso a los programas de educación no formal [2].

Mejía [3] indica que una de las tendencias en la investigación del aprendizaje, derivada de las actividades que caen dentro de la educación no formal, es el aprendizaje en espacios cuyo propósito es la difusión de la ciencia y la cultura. Ejemplo de estos espacios son los museos, zoológicos, centros tecnológicos, galerías de arte, entre otros. Estos espacios están caracterizados por formas de interacción libres y variadas que brindan condiciones favorables al aprendizaje.

La interacción espontánea que se da en museos y espacios de divulgación de la ciencia y la cultura, es un reflejo de la interacción cotidiana de los participantes en su comunidad ya que los visitantes eligen cómo abordar las actividades y exhibiciones de acuerdo a la comunidad de la que forman parte. Es importante hacer notar que la interacción entre las personas alrededor de los temas y la información de las exhibiciones ofrece una gran oportunidad para el aprendizaje, así como la autoregulación y autodirección del aprendizaje; es decir, estos espacios permiten una participación libre, voluntaria, graduada y dirigida por el mismo participante. De esta manera, los participantes pueden enfocarse en la información y la actividad que les interesa y avanzar a su propio ritmo. Por todo ello, hoy es aceptado que los museos interactivos pueden proporcionar escenarios para propiciar modos alternativos de aprendizaje por medio del contacto directo con objetos, y por la interacción en actividades interesantes y divertidas.

\section{La Experiencia del Usuario}

\subsection{El Enfoque de la Experiencia del Usuario}

Nielsen y Norman [4] hablan sobre la necesidad de distinguir experiencia del usuario y usabilidad, considerando que la usabilidad es un atributo de calidad de una interfaz de usuario asociada a la facilidad de aprender a usar un sistema, su uso eficiente y placentero, mientras que la experiencia del usuario es un concepto más amplio que involucra el análisis de la experiencia de interacción más allá de la relación entre usuario y producto. Por su parte, la definición que ofrece la ISO sobre experiencia del usuario se enfoca en las percepciones de una persona y las respuestas sobre el uso o uso anticipado de un producto, sistema o servicio [5]. 
Desde el punto de la interacción humano-computadora, la experiencia del usuario es un tipo específico de experiencia que considera un mediador particular, los productos interactivos [6].

\subsection{Diseño de la Experiencia del Usuario}

En [7] se define el diseño de la experiencia del usuario como la creación y sincronización de elementos que afectan la experiencia del usuario con la intención de influenciar sus percepciones y comportamiento. Los elementos que se consideran incluyen cosas que el usuario puede tocar, escuchar u oler. También incluye las cosas con las que el usuario puede interactuar, más allá de la interacción física, como las interfaces digitales y las personas que están involucradas. Como se puede apreciar, esta noción del diseño de la experiencia del usuario hace hincapié en la habilidad de integrar los elementos que afectan y enriquecen la experiencia en diferentes sentidos.

Así, para diseñar una experiencia del usuario exitosa los productos deben tomar en cuenta los objetivos del negocio del proyecto, las necesidades de los usuarios del producto y cualquier limitación que pueda afectar la viabilidad de las características del producto.

\subsection{Evaluación de la Experiencia del Usuario}

A través de la evaluación de la experiencia del usuario se puede determinar si el producto está produciendo una experiencia placentera para el usuario, existen diversos enfoques de la evaluación, pero la mayoría de ellos se centra en la identificación de los estados emocionales, ya que estos influencian procesos cognitivos como la capacidad de atención, memorización, rendimiento del usuario y, en general, su valoración del producto.

Bajo este contexto, el grupo que colabora en "All About $U X$ " ha recolectado y clasificado más de 80 métodos para evaluar la experiencia del usuario [8]. En [9] se habla de la evaluación de la experiencia del usuario en los videojuegos y su relación con los estados emocionales. Desde su punto de vista la evaluación de la experiencia del usuario basada en cuestionarios puede ser complementada con el uso de mediciones fisiológicas, ya que al enfoque tradicional de detección de estados emocionales se han ido incorporando otros canales fisiológicos y no fisiológicos.

\subsection{Aprendizaje no Formal a través del Diseño de la Experiencia del Usuario}

En la actualidad, la mayoría de las investigaciones donde se haya utilizado las TIC como apoyo para el aprendizaje no formal, se reporta el uso de estrategias de interacción basadas en juegos o videojuegos con objetivos educativos. Schwartz y Bransford [10] llaman a este enfoque, "preparación para el aprendizaje futuro". Su propuesta indica que las experiencias de aprendizaje significativo, como las que se busca construir a través de las experiencias de los juegos, pueden tener mayor impacto en el participante porque son planteadas en un contexto concreto, que ayuda a que tenga sentido para él. 
Si bien este enfoque favorece que el tema que trata el interactivo sea explorado de una forma más lúdica y, junto con las estrategias de la experiencia del usuario, se espera que logren despertar el interés por parte de los usuarios, el aprendizaje del tema puede ser reforzado por a través del contexto del usuario. Este planteamiento puede verse con más claridad en los museos interactivos, donde los sistemas interactivos se acompañan de entornos que permiten apoyar los temas desarrollados en cada una de las exhibiciones.

\section{Diseño de la Experiencia del Usuario en Espacios Interactivos de Aprendizaje no Formal}

Como se mencionó en la sección anterior uno de los puntos centrales al diseñar un espacio interactivo orientado al aprendizaje no formal es crear una experiencia adecuada a los objetivos de aprendizaje. En la propuesta presentada en este documento consideramos que para evaluar la experiencia del usuario de un espacio interactivo es necesario integrar en la metodología de diseño el enfoque de experiencia del usuario. En esta sección se indica cómo hemos adaptado nuestro proceso de diseño de software interactivo para crear una experiencia del usuario en espacios interactivos de aprendizaje no formal.

\subsection{Metodología General para el Diseño de la Experiencia del Usuario en Espacios Interactivos}

La metodología utilizada por el grupo ESIE para desarrollar software interactivo plantea las siguientes fases generales: (1) Planteamiento, (2) Diseño, (3) Realización (4) Pruebas y evaluación, (5) Cierre, (6) Retroalimentación y mantenimiento. Esta metodología se basa en el proceso de diseño centrado en el usuario, por lo una parte fundamental es el análisis de las necesidades de los usuarios del software interactivo. Este análisis puede será complementado usando el enfoque de diseño de la experiencia del usuario. En esta sección se describe cómo se propone complementar esta metodología incorporando el enfoque de la experiencia del usuario.

Planteamiento. Esta fase se definen los objetivos de aprendizaje, quién es la audiencia y principalmente se trata de entender las necesidades del cliente. El trabajo con los expertos del tema y el aprendizaje es central, el equipo se debe empezar a familiarizar con los contenidos así como con las estrategias que se utilizan para favorecer el aprendizaje. Cuando resolvemos nuestro problema desde la visión del diseño de la experiencia del usuario, las estrategias de aprendizaje son una referencia para elegir la forma en que se plantea una actividad pero también deberá analizarse si las actividades se pueden hacer de forma lúdica.

Otro elemento central es conocer el contexto en el que se usará el espacio interactivo. Para crear la experiencia del usuario es fundamental considerar el contexto, como una herramienta para el refuerzo de la experiencia que se está construyendo. Por ejemplo, si es un espacio bajo el auspicio de un patrocinador se debe tomar en cuenta 
la imagen del patrocinador, si es para un museo se debe considerar el papel que tiene el espacio dentro de la sala del museo y la imagen misma del museo.

Diseño. En esta fase se define el concepto central del espacio de interacción, se propone la historia y el estilo de diseño. Esta es una fase central para la definición de la experiencia del usuario, ya que se debe definir una historia que sea capaz de captar la atención de los usuarios y logre integrar los objetivos de aprendizaje de una forma natural. De acuerdo con Schell 11, el diseño de una buena experiencia ocurre cuando se observa el juego desde distintas perspectivas. En su libro "The art of game design: a book of lenses" plantea 100 lentes para apoyar el diseño de un juego, cada uno de estos lentes guía el diseño a través de una serie de preguntas sobre el diseño del juego.

La idea general de la experiencia propuesta se plantea al cliente a través de un guión conceptual y un primer prototipo que pueda representar la idea. El guión conceptual debe especificar los objetivos de aprendizaje planteados así como la estrategia utilizada para favorecer el aprendizaje.

Realización. En esta fase se crean los detalles del juego, las reglas, los retos y la relación con los objetivos de aprendizaje. Dependiendo de las necesidades específicas de cada espacio interactivo, se buscará que los objetivos de aprendizaje estén asociados con los avances en la historia, que se incluyan retos que mantengan la atención de los usuarios y que la interacción produzca una retroalimentación que refuerce la experiencia que se diseñó.

Se elige una parte representativa del concepto para desarrollarse con el detalle suficiente para hacer una prueba del espacio propuesto.

Pruebas y evaluación. El nuevo prototipo del espacio interactivo se pone a prueba con usuarios finales. En el enfoque de evaluación de la usabilidad se observa y analiza cómo un grupo de usuarios reales utiliza un sistema interactivo, adicionalmente, en el enfoque de experiencia del usuario se deberá conocer la percepción del usuario, para ello se pueden utilizar diferentes instrumentos.

El experimento estará controlado por el evaluador en un laboratorio de usabilidad y deberá tratar de reconstruir el contexto real del espacio interactivo, ya que la experiencia no estará completa sino se tiene el contexto adecuado.

A partir las observaciones realizadas en esta evaluación, se retroalimenta al equipo y se hacen las correcciones correspondientes en una nueva fase de realización. 
Cierre. La fase de cierre se gestiona la entrega del producto, incluyendo manuales de uso, instalación y las condiciones de uso que favorecerán la experiencia creada.

Retroalimentación y mantenimiento. Cuando el producto se ha entregado y ha estado en uso, nuevamente se analiza su funcionamiento y determinan las modificaciones que se deben realizar de acuerdo a la experiencia planteada originalmente.

\subsection{Diseño y Evaluación de la Experiencia del Usuario para un Espacio Interactivo Orientado a la Experimentación Musical “¿A qué Suena la Vida?"}

Para ilustrar la aplicación de nuestra propuesta para el desarrollo de espacios interactivos describiremos un caso concreto donde se construyó y evaluó una experiencia específica.

Planteamiento. La empresa "Siete Colores, Ideas Interactivas", solicitó el desarrollo del espacio interactivo "¿A qué suena la vida?” que forma parte de las exhibiciones del museo "La Rodadora" ubicado en Ciudad Juárez, Chihuahua. Los temas principales que abordan en el museo son: entorno natural, donde se presenta información sobre las características geológicas, climáticas y la biodiversidad de la región; entorno social, en este espacio se retoma la vida social de Ciudad Juárez, también se busca fomentar la expresión y reflexión para promover la cooperación y la participación de la comunidad. Estos temas definen las dos secciones más importantes del museo.

La exhibición “¿A qué suena la vida?” pertenece a la sección de entorno social y tiene como objetivo proporcionar un espacio lúdico colaborativo que permita a niños de ocho años en adelante participar en un ensamble musical y simular experiencias básicas de una sesión de grabación usando guitarras eléctricas y una consola de efectos sonoros.

El espacio designado para la exhibición tiene forma cilíndrica con tres metros de diámetro. El espacio interactivo está diseñado para niños de 8 años en adelante.

El objetivo general del espacio interactivo es el desarrollo de un espacio musical interactivo en modo colaborativo siguiendo el enfoque de los juegos rítmicos [12].

De manera más específica se plantean los siguientes puntos:

- Desarrollo de un espacio interactivo y colaborativo en donde se simulen experiencias básicas de un ensamble musical.

- Crear un espacio de experimentación lúdica musical.

- Desarrollar una sesión de grabación de un tema musical. 
Diseño de la experiencia del usuario. El reto más importante para los usuarios es ejecutar una sesión de ensamble musical donde su participación esté acorde con un género musical y que la participación de todos sea congruente con el resto de las intervenciones. El desarrollo de estas habilidades requiere práctica individual y en conjunto. En el caso de este interactivo se plantea guiar a los participantes de forma grupal a través de una melodía base y con información visual en el espacio interactivo, así como sincronizar de forma automática las ejecuciones individuales para que sean congruentes musicalmente entre sí.

Adicionalmente, se indica que escuchamos la melodía pero sentimos el ritmo [13], a partir de esta percepción la gente mueve sus cuerpos al ritmo de la música. Este movimiento involucra percepción de la posición del cuerpo, percepción del movimiento y balance, percepción visual y auditiva. La capacidad de sentir e interpretar los tiempos fuertes de patrón rítmico permite que las personas se muevan y reacciones al tiempo de la música. Por lo general los pulsos fuertes de un patrón rítmico se ejecutan más fuerte, son más largos o presentan ambas características, y la estructura métrica (la que te hace moverte) se deriva de estos pulsos.

Esta revisión de las temáticas del aprendizaje y percepción del ritmo en los niños nos da un marco para el diseño de una experiencia de usuario. De esta forma, el espacio interactivo "¿A qué suena la vida?" deberá ser un espacio atractivo que permita a los visitantes tener una experiencia colaborativa a través de la formación de un grupo o ensamble musical, donde los participantes deberán sentir la experiencia de ser un ídolo musical.

$\mathrm{Al}$ ingresar al espacio interactivo el visitante encontrará cuatro guitarras y una consola de efectos sonoros tipo DJ (figura 1).

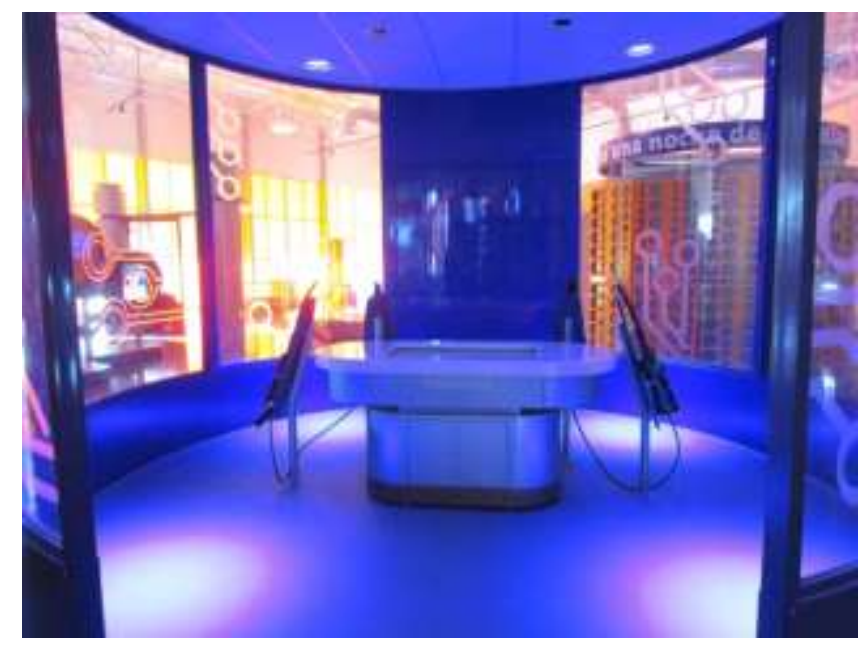

Fig. 1. Espacio interactivo

Realización. Una vez que se define el concepto con el que se trabajará se detallan las características del funcionamiento del espacio interactivo. Se identifica que el público principal de la exhibición es de la región norte de México y que algunos de los géneros musicales más representativos para este público de la zona son: rock, pop y tribal. 
Este análisis nos permite identificar qué géneros musicales serán mejor recibidos por los participantes.

Para reforzar la experiencia de tocar un instrumento se utilizarán dispositivos que simulan una guitarra eléctrica real y una consola de sonidos (figura 1).

Para iniciar la experiencia del usuario, el interactivo se reproduce un mensaje auditivo y visual que invita a los participantes a alcanzar uno de los objetivos del interactivo ser un "ídolo musical" (figura 2). Al finalizar, se apagan las luces y la grabación de la sesión de los participantes se reproduce mientras los usuarios salen del espacio con sonidos vitoreó y aplausos, como sucede al final de un concierto.

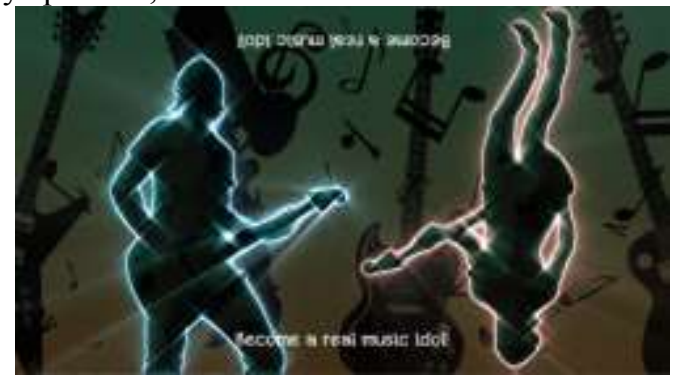

Fig. 2. Mensaje de bienvenida al espacio interactivo

Pruebas y evaluación. De acuerdo con lo presentado en la sección de evaluación de la experiencia del usuario, debemos tratar de obtener la percepción del usuario sobre la experiencia planteada. Para este espacio interactivo se desarrolló un prototipo del interactivo y se realizaron pruebas con usuario. En la sesión de evaluación participaron 21 usuarios en grupos de 3. En cada sesión de evaluación, se grabó la interacción, reacciones y comentarios de los usuarios.

A partir de los videos de las sesiones de evaluación se pueden analizar las actividades de los usuarios e identificar sus estados afectivos y de los usuarios al realizarla. Por ejemplo, se solicita a los participantes que tienen guitarra que empiecen a probarla y al participante que está frente a la consola pruebe los botones de la misma.

A partir de los videos se observa lo siguiente:

- La mayoría de los usuarios tomaron la guitarra se la colocaron y de inmediato intentaron presionar los botones de la misma para poder generar algún sonido.

- Los usuarios que utilizaron la consola de DJ, se quedaron desconcertados, la mayoría de los usuarios no identificaron si ellos generaban algún sonido aunque si presionaban los botones de la consola.

Una vez identificada la reacción de desconcierto del usuario y que no pudo realizar la actividad debemos buscar una estrategia de diseño que nos ayude a mejorar la experiencia. De acuerdo con las guías que proporciona Schell, el lente de "lo básico de la experiencia" nos permite identificar que lo básico de la experiencia de la consola de DJ es agregar efectos de sonido sobre la ejecución de todos los participantes, mientras que la consola de DJ es un instrumento muy específico que no es tan fácil de usar por una persona que no ha tenido un entrenamiento previo (figura 2), así que es necesario rediseñar la consola por una más simple pero que cumpla con los objetivos del espa- 
cio, es decir, un instrumento que permita que el participante se integre a la creación musical (figura 3).

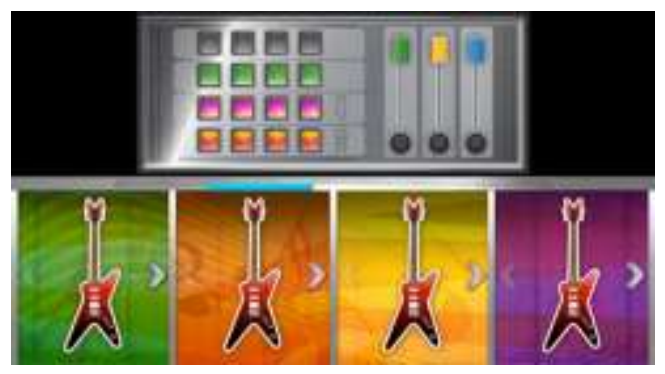

Fig. 3. Interfaz de usuario con consola de DJ realista

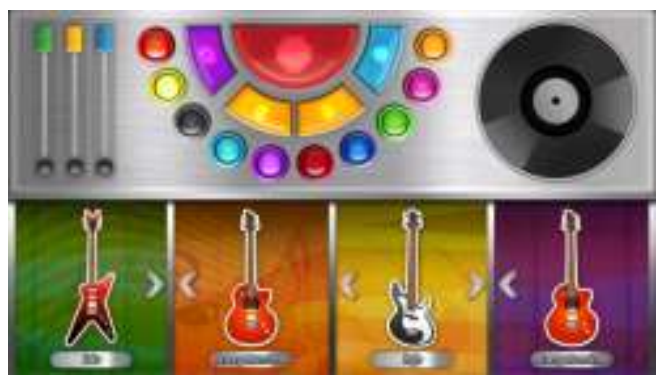

Fig. 4. Interfaz de usuario con consola de DJ rediseñada

La identificación del estado afectivo también puede ser usada para apoyar el análisis de las respuestas en los cuestionarios de salida. Una de las preguntas que se hicieron fue "¿volverías a jugar este juego?", hubo algunos participantes que contestaron que no, o bien que si pero que ahora quisieran "tocar música" refiriéndose al uso de las guitarras. $\mathrm{Al}$ analizar el estado emocional de los participantes que indicaron que no volverían a jugar o lo harían siempre y cuando usen una guitarra, observamos que en la sesión donde participaron les tocó usar la consola de DJ y la experiencia no les fue placentera.

Estos resultados nos indican que la experiencia del usuario de la consola de DJ no es la adecuada, no es suficiente clara, no es divertida y por lo tanto no es placentera. Así que fue necesario rediseñar esta parte del espacio interactivo.

\section{Conclusiones}

En esta propuesta se plantea que un espacio interactivo que busque apoyar el aprendizaje no formal debe estar diseñado pensando en crear una experiencia del usuario para obtener una mejor aceptación por parte de los usuarios. 
La primera aproximación hacia una metodología de diseño de espacios interactivos de aprendizaje no formal, descrita en este documento, nos permite identificar de mejor forma si nuestro producto interactivo cumple o no con los objetivos de aprendizaje para los cuales fue creado, en contraste con una metodología guiada sólo por la usabilidad, donde sí se puede observar si el producto será usable pero no sabemos si le agradará suficientemente al usuario para que concluya la actividad propuesta y con esto se tengan mayores probabilidades de alcanzar los objetivos de aprendizaje.

Junto con la formalización de la metodología se trabajará en la evaluación de este tipo de espacios, para ello, es necesario contar con instrumentos que nos ayuden a identificar la percepción del usuario cuando interactúa en el espacio, ya sea durante la misma evaluación o en una fase posterior de análisis.

\section{Referencias}

1. Hassan Montero, Y., Martín Fernández, F. J.: La experiencia del usuario. No Solo Usabilidad, 4 (2005).

2. UNESCO: Revision of the International Standard Classification of Education (ISCED), 36C/19, 5 de septiembre de 2011. Conferencia general, 36ava Sesión. París, Francia: Unesco (2011).

3. Mejía, R.: Tendencias actuales en la investigación del aprendizaje informal. Revista Sinéctica, 26, 4--11 (2005).

4. Nielsen, J., Norman, D.: The Definition of User Experience. http://www.nngroup.com/articles/definition-user-experience/, consultado: 7 de marzo de 2014 (2014).

5. ISO 9241-210: Human-centred design process for interactive systems (2007).

6. Hassenzahl, M.: User Experience and Experience Design. En: Soegaard, Mads y Dam, Rikke Friis (eds.). "The Encyclopedia of Human-Computer Interaction, 2nd Ed.". Aarhus, Denmark: The Interaction Design Foundation (2013).

7. Unger, R. y Chandler, C.: A Project Guide to UX Design: For user experience designers in the field or in the making. Pearson Education (2009).

8. Allaboutux.org: User Experience White Paper « All About UX. Disponible en: http://www.allaboutux.org/uxwhitepaper (2014).

9. Loscetales, A., Cerezo, E., Creatura, M., González, J. L., Minguez, J., Gil, R., Baldassarri, S., López, Y.: Proyecto NeuroGame: Neuro-Evaluación Multimodal de Videojuegos, En Actas del XIII Congreso Internacional de Interacción Persona Ordenador (Interacción 2012), 3-5 octubre 2012, Elche, España (2012).

10. Schwartz, D. L., Bransford, J. D.: A time for telling. Cognition \& Instruction, 16(4), 475-522 (1998).

11. Schell, J.: The Art of Game Design: A book of lenses. CRC Press (2008).

12. Adams, E.: Fundamentals of Game Design. 2a. edición, New Riders (2010).

13. Phillips-Silver, J., Trainor, L. J.: Feeling the Beat: Movement Influences Infant Rhythm Perception. Science, 308 (5727), 1430--1430 (2005) 